\title{
Title: Determination of Optical-Field Ionization Dynamics in Plasmas through the Direct Measurement of the Optical Phase Change
}

\section{Author(s):}

Submitted to:
Antoinette J. Taylor, Fiorenzo G. Omenetto, George Rodriguez (MST-10)

Craig W. Siders, Jennifer L. W. Siders, University of San Diego

C. Downer, University of Texas, Austin

DOE Office of Scientific and Technical Information (OSTI)

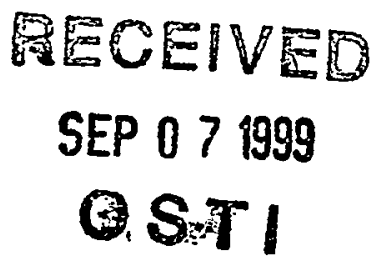

\section{Los Alamos}

Los Alamos National Laboratory, an affirmative action/equal opportunity employer, is operated by the University of California for the U.S. Department of Energy under contract W-7405-ENG-36. By acceptance of this article, the publisher recognizes that the U.S. Government retains a nonexclusive, royaltytree license to publish or reproduce the published form of this contribution, or to allow others to do so, for U.S. Government purposes. Los Alamos National Laboratory requests that the publisher identify this article as work performed under the auspices of the U.S. Department of Energy. Los Alamos National Laboratory strongly supports academic freedom and a researcher's right to publish; as an institution, however, the Laboratory does not endorse the viewpoint of a publication or guarantee its technical correctness. 


\section{DISCLAIMER}

This report was prepared as an account of work sponsored by an agency of the United States Government. Neither the United States Government nor any agency thereof, nor any of their employees, make any warranty, express or implied, or assumes any legal liability or responsibility for the accuracy, completeness, or usefulness of any information, apparatus, product, or process disclosed, or represents that its use would not infringe privately owned rights. Reference herein to any specific commercial product, process, or service by trade name, trademark, manufacturer, or otherwise does not necessarily constitute or imply its endorsement, recommendation, or favoring by the United States Government or any agency thereof. The views and opinions of authors expressed herein do not necessarily state or reflect those of the United States Government or any agency thereof. 


\section{DISCLAIMER}

Portions of this document may be illegible in electronic image products. Images are produced from the best available original document. 


\title{
Determination of Optical-Field Ionization Dynamics in Plasmas through Direct Measurement of the Optical Phase Change
}

\author{
Antoinette J. Taylor*, Fiorenzo G. Omenetto, George Rodriguez, MST-10 \\ Craig W. Siders, Jennifer L. W. Siders, University of San Diego \\ Michael C. Downer, University of Texas, Austin
}

\begin{abstract}
This is the final report of a three-year Laboratory Directed Research and Development (LDRD) Project at Los Alamos National Laboratory (LANL). The detailed dynamics of an atom in a strong laser field is rich in both interesting physics and potential applications. The goal of this project was to develop a technique for characterizing high-field laser-plasma interactions with femtosecond resolution based on the direct measurement of the phase change of an optical pulse. We developed the technique of Multi-pulse Interferometric Frequency Resolved Optical Gating (MI-FROG), which recovers (to all orders) the phase difference between pumped and unpumped probe pulses, enabling the determination of sub-pulsewidth time-resolved phase and frequency shifts impressed by a pump pulse on a weak probe pulse. Using MI-FROG, we obtained the first quantitative measurements of high-field ionization rates in noble gases and diatomic molecules. We obtained agreement between the measured ionization rates and those calculated for the noble gases and diatomic nitrogen and hydrogen using a one-dimensional fluid model and rates derived from tunneling theory. However, much higher rates are measured for diatomic oxygen than predicted by tunneling theory calculations.
\end{abstract}

\section{Background and Research Objectives}

The interaction of ultrashort, high-intensity laser pulses with solid and gas targets is a field of growing interest. Highly ionized atmospheric-density plasmas are a potential source of coherent VUV and $\mathrm{x}$-ray light, as well as a potential medium for charged-particle acceleration [1]. Creation of such plasmas via ionization by intense, femtosecond pulses holds promise for the precise control of the initial plasma conditions that are critical to these applications. At the same time, new, quantitative experimental diagnostics compatible with

*Principal Investigator, e-mail: ttaylor@lanl.gov 
high gas density and ultrafast time scales are needed to measure the ionization and subsequent plasma dynamics that give rise to these conditions. Furthermore, the interaction of a highintensity, ultrashort pulse with a solid target is of interest both scientifically and as a source of ultrashort x-ray pulses. Again, appropriate diagnostics are lacking to fully understand the physics of these interactions. In particular, for solid targets, the spatial scale length of the electron density gradient in an expanding plasma is difficult to determine accurately, yet essential for the understanding of absorption mechanisms in the ultrafast regime. We describe here a technique for characterizing laser-plasma interactions with femtosecond resolution based on the direct measurement of the phase change of an optical pulse using frequencyresolved optical gating (FROG).

FROG [2-4] is a recently developed ultrafast diagnostic technique to measure the full electric field, both instantaneous amplitude and phase, of an arbitrary ultrashort pulse. Experimentally, this technique involves splitting the pulse to be characterized into two replicas, which then interact in a nonlinear optical medium for a range of delays between the two pulses, giving an autocorrelation-type signal. This signal is then frequency-resolved with a spectrometer for each delay value, producing the FROG trace, $\mathrm{I}_{\mathrm{FROG}}(\omega, \mathrm{t})$. The FROG trace is a spectrogram of the electric field, $\mathrm{E}(\mathrm{t})$, of the laser pulse and it contains essentially all information about the intensity and phase evolution of the pulse. The problem of inverting the FROG trace to find $E(t)$ is equivalent to the two-dimensional phase retrieval problem, a well known, solved problem in the field of image science. Prior to the research described in this project, FROG was being used, in our laboratory and elsewhere, solely as an advanced diagnostic tool for characterizing ultrafast laser systems operating in wavelength ranges spanning the infrared to the ultraviolet. However, because FROG is a general, broadband optical diagnostic technique, we recognized the potential to extend its diagnostic capability in a variety of research areas where the characterization of the full electric field of an optical pulse would provide critical insight into physical processes. This project is based on our original ideas to develop FROG as an optical probing technique to elucidate the physics of femtosecond ionization dynamics in plasmas.

Research in the complete characterization of the electric field of optical pulses is a very "hot " topic in the ultrafast optics community. In the past few years, several groups, 
including our own, have been heavily involved in the development and improvement of coherent diagnostic techniques for complete characterization of an optical pulse. For the most part such techniques have been used primarily to characterize the output of laser systems. The extension of FROG for the characterization of plasmas impacts not only on the field of plasma physics, but also the field of ultrafast science. Our expertise in both coherent ultrafast diagnostics such as FROG and in plasma physics, as well as our state-ofthe-art ultrafast laser facilities, has put us in a unique position to seize this opportunity.

The objective of this project was to develop FROG as an advanced optical probing technique to elucidate the physics of femtosecond ionization dynamics in plasmas. To achieve this goal we optimized FROG to be used in a temporally gated setup to directly probe, with femtosecond resolution, the ionization dynamics in a plasma. We then used this optimized version of FROG to systematically investigate femtosecond field ionization dynamics in atmospheric-pressure noble gases. Further refinement of FROG as a plasma diagnostic, both in terms of experimental technique and interpretation of data, ensued as these experiments progressed. As an extension of these experiments, the field ionization dynamics in atmospheric gases of molecules was also be studied using FROG. The results of the atomic and molecular experiments were compared.

\section{Importance to LANL's Science and Technology Base and National R\&D Needs}

Basic Scientific Impact. The detailed dynamics of an atom in a strong laser field is rich in both interesting physics and potential applications. Ionization-based high-harmonic generation enables efficient and tunable sources of short wavelength femtosecond radiation and may yield the first attosecond pulses. New ultrafast diagnostics are needed to measure the ionization and subsequent plasma dynamics that give rise to these conditions. The plasma diagnostic we developed permits, for the first time, the understanding of the dynamics of short-pulse laser plasmas with femtosecond temporal resolution. Such a diagnostic is important for elucidating the physics of laser-plasma interactions, for measuring ionization rates and recombination rates, and for characterizing nonlinear processes such as stimulated Raman and stimulated Brillouin scattering. It is also essential for pursuing a variety of applications of short-pulse laser plasmas such as VUV recombination lasers, harmonic 
generation, laser-plasma particle acceleration, femtosecond $\mathrm{x}$-ray sources and laser-induced lightning. In all of these applications the initial plasma conditions must be carefully controlled (and hence, characterized) to optimize the process. This diagnostic can also be used to study and optimize the dynamics of short-pulse laser ablation, a technique under development for materials processing and medical applications. It also would be useful for optimizing the performance of a measurement scheme for femtosecond VUV pulses based on field-ionization dynamics.

Moreover, recent observations of self-channeling of high-peak-power femtosecond laser pulses in air have sparked considerable interest because the long-interaction-length, intense laser pulses thus produced have potential applications such as laser-induced lightning, laser-pumped XUV sources, advanced LIDAR sources, and laser-plasma-based accelerators. Weakly focused, 15-mJ, 100-fs pulses have been observed to self-channel into filaments that propagate at intensities approaching $10^{14} \mathrm{~W} / \mathrm{cm}^{2}$ for distances greater than $20 \mathrm{~m}$. The mechanism for this self-channeling is attributed to a balance between self-focusing from the intensity-dependent refractive index and the combined effects of natural diffraction and refraction from the index change due to plasma formation from the intense optical pulse. However, the physics of self-channeling has not been studied experimentally in detail. Our plasma diagnostic based on FROG could be used to understand and optimize this process.

Furthermore, although beyond the scope of this project, we believe that FROG is a technique that can be extended to other research areas where characterization of the full electric field of an optical pulse can provide insight into various physical and chemical processes. Such research areas include materials science, shock physics, physical chemistry, biology, and atomic and molecular physics. For example, development of FROG as a diagnostic technique to characterize the coherent interaction between optical pulses and optical and optoelectronic materials and devices could have an enormous impact in areas such as signal processing, optical communications, and advanced computer systems. These types of measurements should be extremely important to coherent optoelectronic communication systems relying on soliton propagation and/or wavelength division multiplexing, since such systems are sensitive to phase errors induced in the optical signal. Moreover, the development of FROG as a novel dynamic spectroscopic technique to elucidate ultrafast 
processes will find a wide range of applications ranging from biophysics (exploring the kinetics of proteins), to plasma physics (understanding multiphoton ionization processes), to electrical engineering (examining carrier relaxation in semiconductors).

Relevance to LANL and National $R \& D$ Needs. The plasma diagnostic technique based on FROG developed in this project will enable the characterization of the dynamics of laser-plasma interactions with femtosecond resolution. As such, this technique will be useful to LANL researchers in several divisions who routinely study and use laser-plasma interactions in their programs. FROG should also prove a useful diagnostic for plasma physics issues relevant to nuclear weapons programs such as Inertial Confinement Fusion (ICF) and Above Ground Experiments (AGEX). In particular, our FROG plasma diagnostic may be valuable in understanding physics issues relevant to the fast ignitor concept for laser fusion. In the fast-ignitor approach, target ignition is achieved with a high-intensity picosecond laser pulse rather than by hydrodynamic compression, hence eliminating much of the drive energy needed by the ignition process. This fast-ignitor approach could significantly reduce the \$1-billion cost of the proposed National Ignition Facility (NIF). A serious research program is currently underway to explore this concept at the Nova laser facility where chirped pulse amplification of a 0.5 picosecond pulse to the 800 -Joule level has been achieved. Using FROG, we can address one of the critical issues relevant to fast ignitor, the ability of the picosecond ignitor pulse to propagate through a long-scale-length plasma to the interaction region of the target.

Furthermore, because FROG is a general optical diagnostic technique, it should prove a useful diagnostic in a variety of research areas important to the laboratory, such as plasma physics, materials science, shock physics, physical chemistry and atomic and molecular physics. Completion of this project has increased our expertise in FROG; it will have an additional payoff in enhancing our competitive advantage in these other scientific areas. Moreover, the development of FROG as a novel dynamic spectroscopic technique to elucidate ultrafast processes may be useful to a variety of programs Laboratory wide.

Finally, as a result of this project we have initiated or grown collaborations involving FROG with colleagues at Southwest Sciences, Inc, U.C. San Diego, National Institute of Standards, University of Texas and Sandia, thus providing the additional benefit to LANL of 
fostering industrial, university and inter-DOE Laboratory collaborations. Since we have developed FROG as a useful diagnostic tool, we are well poised to attract industrial partners via CRADAs to further develop and apply this technology. Previously, we have collaborated with smaller, scientific instrumentation companies, such as Southwest Sciences, Inc., Positive Light, and Clark Instrumentation, on issues involving the development of commercial FROG devices for the characterization of laser systems. We expect such companies to consider commercialization of the scientifically more powerful diagnostic capability developed in this project. Finally, we note that we have a patent pending on this diagnostic.

\section{Scientific Approach and Accomplishments}

In this project we developed ultrafast pulse characterization techniques, specifically Frequency Resolved Optical Gating or FROG to investigate femtosecond field ionization dynamics in atmospheric-pressure noble gases. These are relatively simple systems to study from an experimental point of view, yet they provide rich physics issues to study such as the relative roles of ionization mechanisms including collisionless tunneling ionization, electron impact ionization and transient resonant enhancements. Furthermore, modeling of the ionization dynamics of such systems, although nontrivial, is relatively straightforward. Therefore, we used this type of experiment to implement and perfect FROG as a plasma diagnostic, both in terms of experimental technique and interpretation of data.

One goal was to determine the evolution of the ionization dynamics in helium, neon, argon, krypton and xenon. For each gas, the pressure was varied up to 1 atmosphere and the intensity of the ionization pulse was varied from the ionization threshold to $>1015 \mathrm{~W} / \mathrm{cm}^{2}$, the upper limit of density and intensity being limited by defocusing of the beam. The details of these ionization dynamics depend on the gas, pressure and pulse intensity and provide insight into the physics of the ionization mechanisms. For conditions of low pressure and low final ionization state, the dominant ionization mechanism is collisionless radiative ionization of each atom through its interaction with the intense light field. We modeled our results from this regime with a strong-field tunneling theory using both Keldysh [5] and Ammosov [6] formulations and compared these theoretical results with our data. 
For these measurements we used a moderate-energy, high-repetition-rate Ti:sapphire system that yields $1-\mathrm{mJ}, 180-\mathrm{fs}, 800-\mathrm{nm}$ laser pulses at a kilohertz repetition rate. For initial measurements a pump-probe experimental set-up was implemented by splitting the input laser beam into a weak probe beam and an orthogonally polarized, energetic pump beam, and delaying the probe beam temporally with respect to the pump beam. The pump beam was focused to intensities around $10^{14} \mathrm{~W} / \mathrm{cm}^{2}$ into a cell containing a noble gas at a pressure of one atmosphere or less to ionize the gas. The time-delayed probe was focused through the cell such that it spatially overlapped the pump beam and traverses the ionized gas. The change in the probe's optical phase and amplitude induced by the ionized gas was measured using two single-shot FROG devices: one sampling the pulse before the plasma and one sampling it after it traverses the plasma. The evolution of the ionization dynamics was determined with $<100$-fs resolution by scanning the probe delay line so that the probe first precedes and finally trails the pump pulse and taking FROG traces at each step. Additional diagnostics to measure the pump energy and to characterize the focal region of the pump beam were used. Determination of the probe's electric field phase and amplitude from the FROG traces was accomplished using a previously developed algorithm.

The results of these experiments using this initial approach [Pub. 1] were of limited value for the following reasons. There are well-known "trivial ambiguities" inherent in FROG which prevent the determination of the constant and linear terms in the frequency-dependent phase of the optical field under measurement, $\mathrm{E}(\mathrm{t})$. These two ambiguities are indeed unimportant when characterizing a single pulse in terms of its temporal and spectral intensity profiles as well as its time-resolved center frequency (i.e. its chirp). However, in the case of measuring optical properties of materials, specifically plasmas, these two ambiguities, a delay of the pulse in time and a shift of the electric field oscillations with respect to the intensity envelope, are the lowest-order and therefore often the dominant effects. To illustrate this point, we can define a characteristic length of material $\mathrm{L}_{\varphi}=\pi \mathrm{c} / \mathrm{n} \omega$ where $\mathrm{c}$ is the speed of light, $\mathrm{n}$ the refractive index, and $\omega$ the pulse's center frequency, as that length which produces a phase change of $\pi$. Similarly, $\mathrm{L}_{\Delta}=c \tau / \mathrm{n}$, where $\tau$ is the pulse's temporal width, is that length of material that produces a temporal delay on the order of a pulsewidth. Finally, $L \tau=\tau / k 2$, 
where $\mathrm{k}_{2}$ is the material's group velocity dispersion, is the characteristic length needed to broaden the pulsewidth by a factor of two. For a typical laser-produced plasma, the ratios $\mathrm{L}_{\varphi}$ $: \mathrm{L}_{\Delta}: \mathrm{L} \tau$ are approximately $1: 50: 3000$. Clearly, in the sense of requiring less material path length, sensitivity to changes in the constant and linear phase are needed for plasma dynamics experiments.

Spectral Interferometry (SI, [7]) is a well-known technique for measuring differences in both the zeroth (i.e. a constant) and first order (i.e. time delay) terms of the spectral phase. It has been used to measure time-resolved Langmuir oscillations in plasmas as well as expansion velocities of laser-excited surfaces. A heterodyne technique, SI uses two temporally separated pulses colinearly input to a spectrometer so that they will interfere in the frequency domain yielding a cross term proportional to the phase difference $\cos \varphi(t)$ between the signal and reference beams. In the case of pulses with significant structure in the wing, pre- or post-pulses, such as those derived from amplified laser systems, SI cannot be readily used because all components of the pulse (ASE, wing, pre- and post-pulses) will produce high-contrast fringes with identical fringe spacing. When a small time-domain phase shift is impressed only upon the main pulse, the corresponding SI interferogram is then a complicated admixture of shifted and unshifted fringes that is difficult to interpret.

FROG, on the other hand, utilizes an ultrafast optical gate and long-time-scale integration (e.g. the multi-ms diode array or CCD integration time) and hence is a form of ultrafast boxcar, or gated integrator, detection. It effectively eliminates extraneous long-timescale contributions to the measured spectra. What is required is to study plasma dynamics is a combination of FROG and SI that retains the key ingredient in FROG: the ultrafast optical gate. We developed a combination of standard FROG with multi-pulse spectral interferometry termed Multi-pulse Interferometric FROG (MI-FROG) for the purpose of measuring pump-induced phase changes in materials [Pub. 2, 3, 8]. This technique uses two pulse trains (with the pulse separations sufficiently large that they do not overlap temporally) for the gate field and the probe field. Note that the two temporally separated pulses that make up the gate pulses are identical, while the two pulses that make up the probe field, though initially identical, will be different due to the effect of an intense pump 
pulse on the probe. An illustrative MI-FROG spectrogram is shown in Figure 1. Cross phase modulation from a co-propagating pump pulse in a nonlinear material results in a redshift/blueshift on the leading/trailing edge of the second pulse [Pub. 6].

The unique abilities of MI-FROG center around its inherently differential nature. Ultrafast changes in the time-domain phase of a laser pulse are measured, unlike standard FROG, to all orders while shot-to-shot fluctuations in the pulse structure are discriminated against. Additionally, a time-domain phase shift can be measured, unlike SI, in a single shot over a time-scale much longer than the pump pulse that induces it. The ultrafast boxcar advantage of FROG is retained in MI-FROG: the sub-picosecond optical gating essentially eliminates amplifier leakage, ASE, satellite pulses, and target emission. The real-time nature of MI-FROG allows immediate and direct observation of pump-pulse time scale dynamics without computational analysis, a significant advantage over traditional single-pulse FROG. Finally, iterative computational techniques [Pub. 2, 7] similar to those used in standard FROG can be used to elucidate femtosecond time-scale detail from the MI-FROG trace.

With our new MI-FROG diagnostic technique in hand we continued our investigation of femtosecond field ionization dynamics in atmospheric pressure noble gases [Pub. 5]. The set-up (shown in Figure 2) for these experiments was as follows. A standard chirped pulse amplified laser system was used to provide 1-mJ, 175-fs, 802-nm pulses at one $\mathrm{kHz}$. Twenty percent of this pulse was used to generate, in a Michelson interferometer, the multi-pulse weak probe and gate sequences while the remainder was frequency doubled in a $0.3-\mathrm{mm} \mathrm{BBO}$ crystal, providing a $30-\mu \mathrm{J}, 125-\mathrm{fs}, 401-\mathrm{nm}$ pump pulse. The pump pulse polarization was either parallel or perpendicular to the probe. After dumping the residual IR, the UV pump and attenuated IR probes were colinearly focused into a backfilled gas cell with lownonlinearity $\mathrm{CaF}_{2}$ windows. Temporal overlap of the pump in the focal region was established via sum frequency generation in the gases, while spatial overlap was ensured using focal plane imaging into a $\mathrm{CCD}$ camera. The gate pulses traveled a separate path to a modified FROG apparatus while the probes were dichroically separated from the pump and entered the same FROG apparatus. The FROG apparatus used a polarization gate via a Kerr nonlinearity. Data was taken with various noble and diatomic molecular gases in the cell at pump-probe increments of $12 \mathrm{fs}$. For each pump-probe delay, three power-spectra and one 
optically gated frequency-resolved spectra (see Figure 3) per each of four CCD images (pumped and unpumped cases with one or two probe pulse) were acquired, thus providing simultaneous and self-consistent dual-channel power spectra and frequency domain interferograms, MI-FROG, and FROG data. Completely automated, one data set consisting of 51 delay points and $255 \mathrm{CCD}$ images was acquired in 30 minutes.

The power spectra channels in Figure 3 allow both centroid-based power spectrum analysis and interferogram analysis, which provide a pulse-width averaged measure of frequency and phase shift, respectively. They can depend sensitively on the detailed temporal and frequency-domain structure of the probe pulse. As MI-FROG recovers, to all orders, the phase difference between the pumped and unpumped probe pulses, we can utilize it to a) verify the intensity and phase structure of the probe pulse and b) recover the subpulsewidth time-resolved phase and frequency shifts impressed upon the probe by the pump pulse and ionization front, independently of the probe pulse structure.

Figure 4a illustrates the doubly time-resolved probe pulse frequency shift in the case of above-threshold ionization of Xenon. The upper, redshifted, diagonal corresponds to $\mathrm{n}_{2} \mathrm{I}$ cross-phase modulation via the neutral gas and is strongest for early pump delays. This asymmetry suggests defocusing is significant, a hypothesis supported by independent probe spectral data. The lower, blue-shifted diagonal is predominately due to the ultrafast ionization front, though comparison of the $p$ - and $s$-polarized cases indicates that neutral gas contributions are not always negligible. Figure $4 \mathrm{~b}$ similarly illustrates the corresponding probe pulse intensity profile versus time. It should be stressed that by using MI-FROG a zero of time is well defined and the apparent motion of the pulse profile is genuine.

Modeling [8] of the data in Figure 4 and similar cases reveal frequency mixing via the transverse plasma current [9] on such a large scale that ultrafast depletion of the probe pulse occurs within the ionization front. Though this mixing is clearly accompanied by $2 \mathrm{D}$ effects not included in the model, the observed ultrafast depletion, as it occurs only within the ionization front and for both polarizations, cannot be due to defocusing or neutral gas effects. We conclude, then, that this work [Pub. 9] represents the first direct, ultrafast time-resolved observation of this phenomenon and the apparent pulse motion in time due to the nonlinear 
interaction. This effect could provide an interesting new route to nonlinear pulse shaping via rapid time-domain modulation

The effect upon a short optical pulse co-propagating a distance $z$ with an ionization front can be viewed in the frame of the pulse as a simple time-dependent phase shift: $\varphi(t)=$ $\left(z \omega_{0} / c\right)\left\{1-n / 2 n_{c r}\right)$ where $n(t)$ is the electron density of the plasma, $n_{c r}=\pi r_{e} \lambda^{2}$ is the critical density of the plasma, $r_{e}$ is the classical electron radius, and $\lambda$ is the center wavelength of the pulse. Since the instantaneous frequency of the pulse is proportional to the derivative of the phase, the pulse will experience an ultrafast time-dependent frequency shift: $\Delta v(t)=\left(\mathrm{zr}_{\mathrm{e}} \lambda / 2 \pi\right) \partial \mathrm{n}(\mathrm{t}) / \partial \mathrm{t}$. Since the plasma density scales linearly with the ambient gas density $\rho$ and laser wavelength, we can normalize this shift by $\rho \lambda$. We can also include the effects of cross-phase modulation in the gas by adding a term proportional to a per-molecule nonlinearity $\gamma_{\mathrm{a}}$ :

$\Delta v(\mathrm{t}) /\left(\mathrm{r}_{\mathrm{e}} \lambda \rho\right)=\mathrm{z}_{\mathrm{g}} \partial\left(\gamma_{\mathrm{a}} \mathrm{I}(\mathrm{t})\right) / \partial \mathrm{t}-\mathrm{z}_{\mathrm{p}} \partial \mathrm{N}_{\mathrm{a}}(\mathrm{t}) / \partial \mathrm{t}$

where $I(t)$ is the pump-pulse intensity, $N_{a}(t)$ is the per-molecule ionization fraction, and $z_{p}$ and $z_{\mathrm{g}}$ are interaction lengths for the plasma and gas respectively. We can see from this equation that an increasing electron density results in a frequency shift to the blue, while for $\gamma_{a}>0$, cross-phase modulation produces a frequency shift towards the red on the leading edge $(\partial \mathrm{I} / \partial \mathrm{t}>0)$ of the pump and a blueshift on the trailing edge $(\partial \mathrm{I} / \partial \mathrm{t}<0)$ of the pump.

To quantitatively compare the measured ionization-front blueshifts with existing models of atomic ionization, we used a reduced frequency-shift parameter $\delta_{v}$ given by $\Delta v / \mathrm{r}_{\mathrm{e}} \lambda \mathrm{N}_{\text {gas }}$, where $\Delta v$ is the measured shift, $\mathrm{r}_{\mathrm{e}}=2.8 \times 10^{-13} \mathrm{~cm}$, and $\lambda$ the laser wavelength. Thus, $\delta_{v}$ is determined solely by the product of the single-atom ionization rate and an interaction length. Figure 5 presents the measured $\delta_{v}$ divided by the confocal parameter. For those cases where the confocal parameter well approximates the plasma length (evidenced by no pump or time-resolved probe absorption and by $\mathrm{n}_{2} \mathrm{I}$-like features in the frequency-shifts), we find good agreement between the measured ionization rates with those calculated with a 1D plasma fluid model and Ammosov ionization rates [8]. Using Keldysh rates yielded ten 
times smaller predicted rates. This data represents the first direct measurement of high-field ionization rates in noble gases. [Pub. 9]

In the case of the homonuclear diatomic gases $\mathrm{H}_{2}$ and $\mathrm{N}_{2}$ (figure 6), we find similar good agreement with the model using published molecular ionization potentials. However, in the case of $\mathrm{O}_{2}$, we find a two order of magnitude disagreement, with tunneling theory overpredicting the ionization rate. Recently, similar disagreement has been observed and an alternative calculation method for the tunneling rate has been proposed. [10]

In conclusion we have developed an advanced optical probing technique to elucidate the physics of femtosecond ionization dynamics in plasmas based on the measurement of the change in the full electric field (phase and amplitude) of an ultrafast optical pulse. To achieve this goal we combined the techniques of Frequency Resolved Optical Gating (FROG) and Spectral Interferometry to directly probe, with femtosecond resolution the ionization dynamics in a plasma. We then used this optimized diagnostic technique MI-FROG to systematically investigate femtosecond field ionization dynamics in atmospheric-pressure noble gases and diatomic molecular gases. MI-FROG allowed the clear identification and differentiation among pump depletion from ionization losses, pump and probe defocusing from the plasma, and the hitherto unseen probe depletion within the ionization front itself.

We performed the first quantitative measurements of high-field ionization rates in noble gases and diatomic molecules. Agreement between the measured ionization rates and those calculated for the noble gases and diatomic nitrogen and hydrogen using a onedimensional fluid model and rates derived from tunneling theory [6] is achieved, while tunneling theory calculations predict much higher rates than measured for diatomic oxygen. 


\section{Publications}

1. Sharp, T., Rodriguez, G., Wood, Wm., and Taylor, A., "Characterization of ultrafast interaction with materials through the direct measurement of the optical phase change," Proceedings of the Society of Photo-Instrumentation Engineers 2701, 229-234 (1996).

2. Kane, D., Rodriguez, G., Taylor, A., and Clement, T., "Simultaneous measurement of two ultrashort laser pulses from a single spectrogram in a single shot," J. Opt. Soc. Amer. B 14, 935-943 (1997).

3. Siders, C., Taylor, A., and Downer, M., "Multipulse interferometric frequency-resolved optical gating: real-time phase-sensitive imaging of ultrafast dynamics," Trends in Optics and Photonics 13: Ultrafast Electronics and Optoelectronics, (Optical Society of America, Washington, DC, 1997). 161-166

4. Siders, C., Taylor, A., and Downer, M., "Multipulse interferometric frequency-resolved optical gating: real-time phase-sensitive imaging of ultrafast dynamics," Opt. Lett. 22, 624-626 (1997).

5. Siders, C., Siders, J., and Taylor, A., "Femtosecond Coherent Spectroscopy at $800 \mathrm{~nm}$ : MI-FROG Measures High-Field Ionization Rates in Gases", in Ultrafast Phenomena XI, (Springer-Verlag, Berlin, 1998) p. 438-440.

6. Rodriguez, G. and Taylor, A. "Measurement of cross-phase modulation in optical materials through the direct measurement of the optical phase change," Opt. Lett. 23, 858-860 (1998).

7. Nicholson, J., Funk, D., Omenetto, F., and Taylor, A., "Evolving FROGs: Phase retrieval from Frequency-Resolved Optical Gating measurements using genetic algorithms," Opt. Lett. 24, (1999), in press.

8. Siders, C., Siders, J. Omenetto, F., and Taylor, A. "Multi-pulse, Interferometric Frequency-Resolved Optical Gating," IEEE J. Quantum Electron., (1999), in press.

9. Siders, C., Siders, J. Omenetto, F., and Taylor, A., "Direct Measurement of High-Field Ionization Rates in Noble Gases," Phys. Rev. Lett., (1999), submitted. 


\section{References}

[1] Gavrila, M., ed., Atoms in Intense Laser Fields, Academic Press: Boston, 1992.

[2] Kane, D., and R. Trebino, R., "Single-shot measurement of the intensity and phase of an arbitrary ultrashort pulse by using frequency-resolved optical gating," Opt. Lett. 18, 823-825 (1993).

[3] Trebino, R., DeLong, K., Fittinghoff, D., Sweetser, J., Krumbgel, M., Richman, B., and Kane, D., "Measuring ultrashort laser pulses in the time-frequency domain using frequencyresolved optical gating," Rev. Sci. Instrum., 68, 3277-3295 (1997).

[4] R. Trebino and D. J. Kane, "Using phase retrieval to measure the intensity and phase of ultrashort pulses: frequency-resolved optical gating," J. Opt. Soc. Am. B, 10, 1101-1111 (1993).

[5] Keldysh, L., "Ionization in the field of a strong electromagnetic wave," Sov. Phys. JETP 20, 1307 (1965).

[6] Ammosov, M., Delone, N., and Krainov, V., Tunnel ionization of complex atoms and atomic ions in an alternating electromagnetic field," Sov. Phys. JETP, 64, 1191 (1986).

[7] Piasecki, J., Colombeau, B., Vampouille, M., Froehly, C., and Arnaud, J., "Nouvelle methode de mesure dela reponse impulsionnelle des fibres optiques," App. Opt, vol. 19, 3749-3755, (1980).

[8] Penetrante, B., Bardsley, J., Wood, W., Siders, C., and Downer, M., "Tonization-induced frequency shifts in intense femtosecond laser pulses," J. Opt. Soc. Am. B 9, 2032-2041, (1992).

[9] Brunel, F., "Harmonic generation due to plasma effects in a gas undergoing multiphoton ionization in the high-intensity limit," J. Opt. Soc. Am. B, 7, 521-526, (1990).

[10] Gibson, G., Berquist, K., Li, M., Guo, C., and Nibarger, J., "Anomolous strong-field ionization of oxygen," Technical Digest of the Conference of Lasers and Electro-Optics '98, JTuB7 (1998). 


\section{frequency}

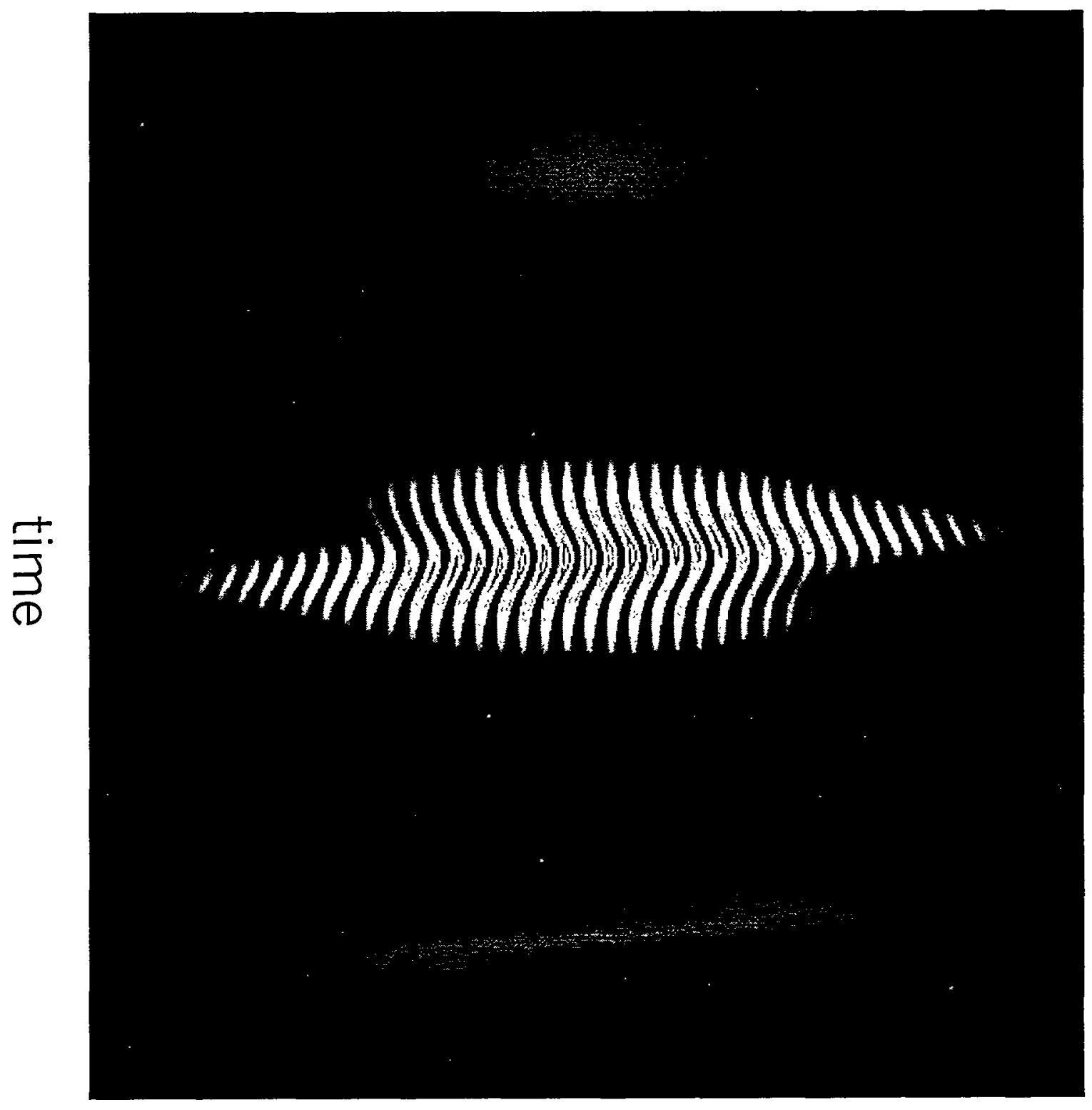

Figure 1. An illustrative calculation of a MI-FROG trace. Time is in units of pump pulse width with the probe two units wide and a pulse separation of 6 units. A cross-phase modulation from a co-propagating pump exists between two individual probe pulses, resulting in a redshift/blueshift on the leading/trailing edge of the second pulse. 


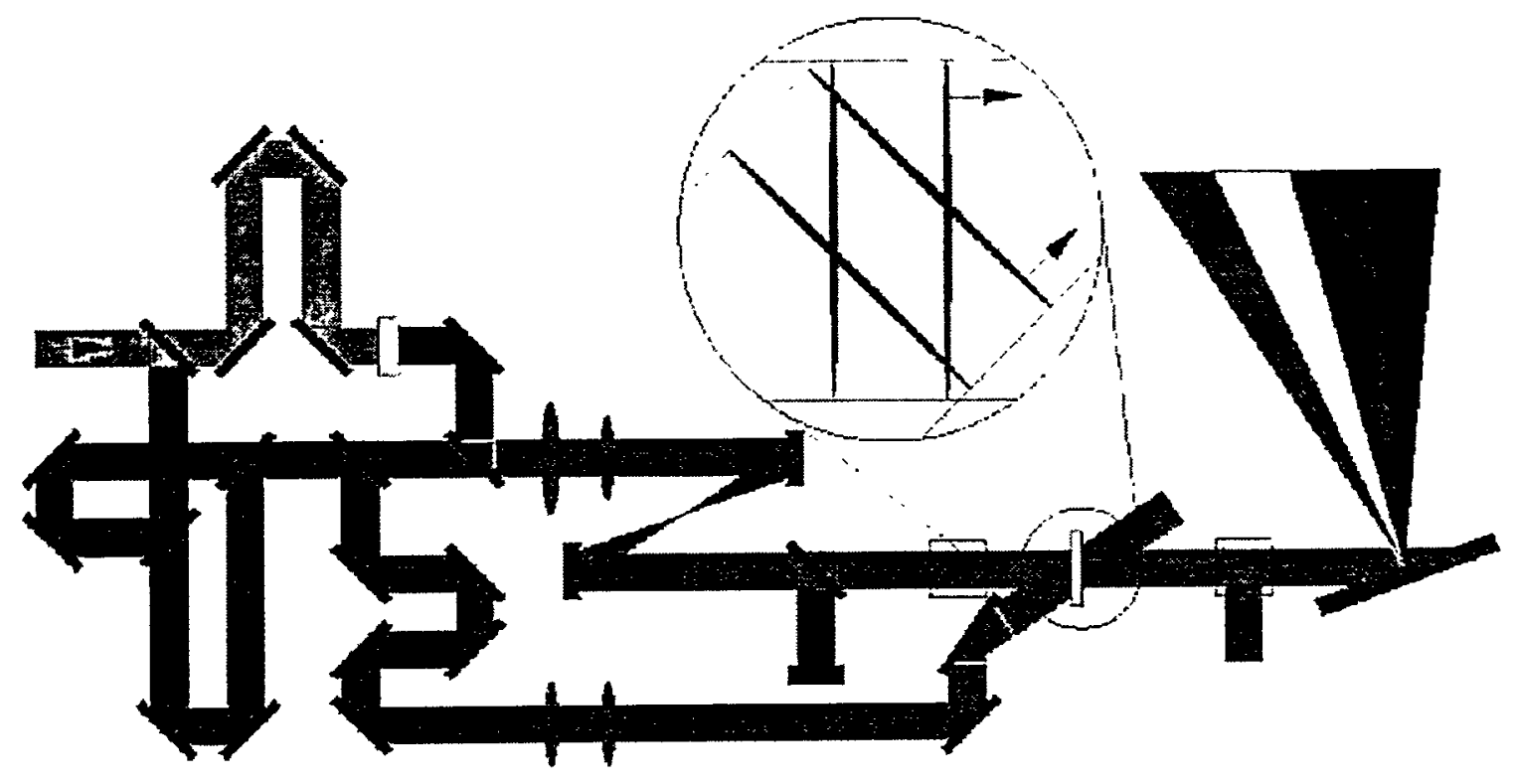

Figure 2. Experimental set-up for the investigation of femtosecond field ionization dynamics using MI-FROG. 


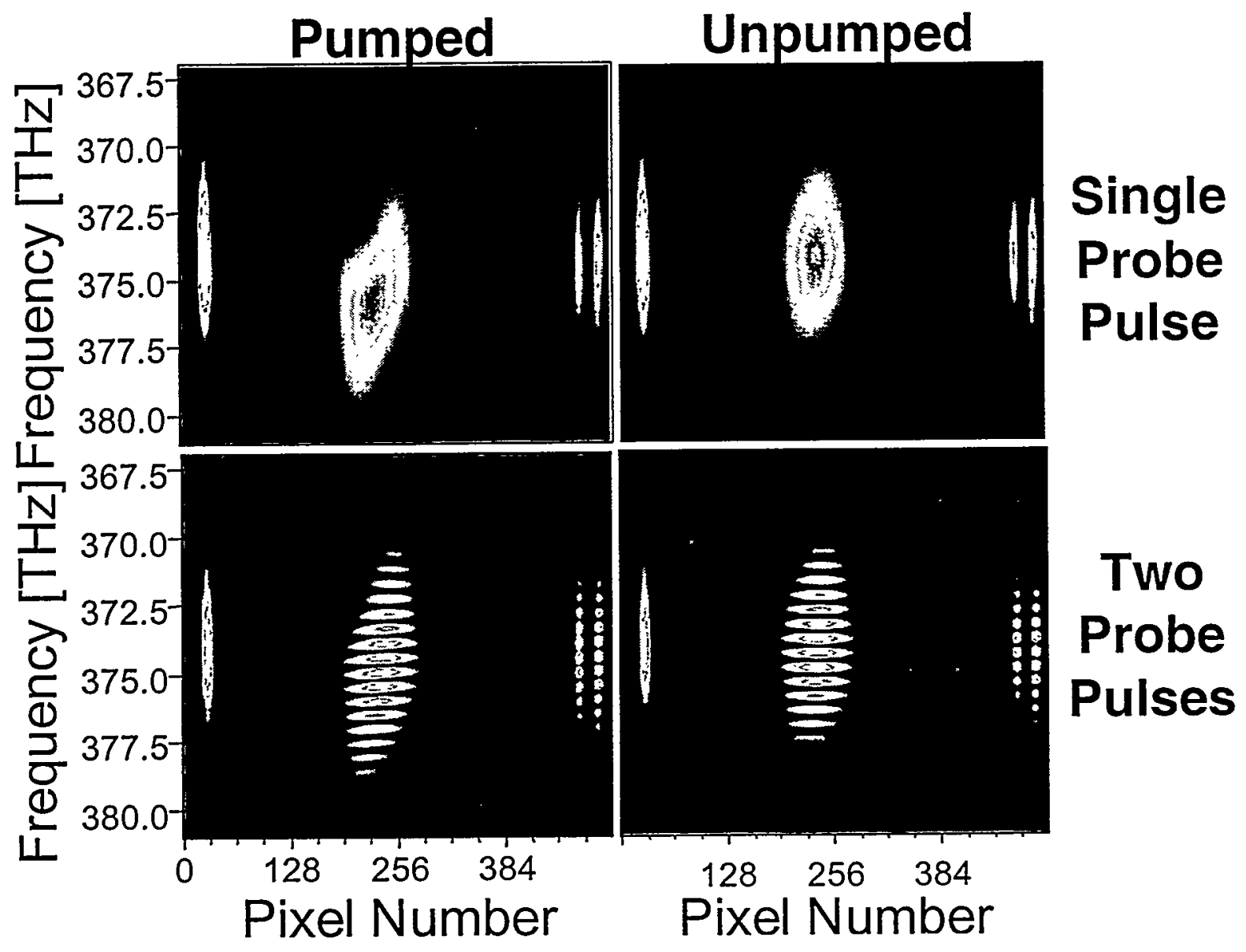

Figure 3. Example CCD data. MI-FROG (bottom) and FROG (top) traces for pumped (left) and unpumped (right) conditions. The three power spectra channels are the incident pulse from the amplifier (left), the single or double pulse sequence entering (right) and exiting the interaction region. Note the strong spectral blueshift and corresponding fringe rotation. 


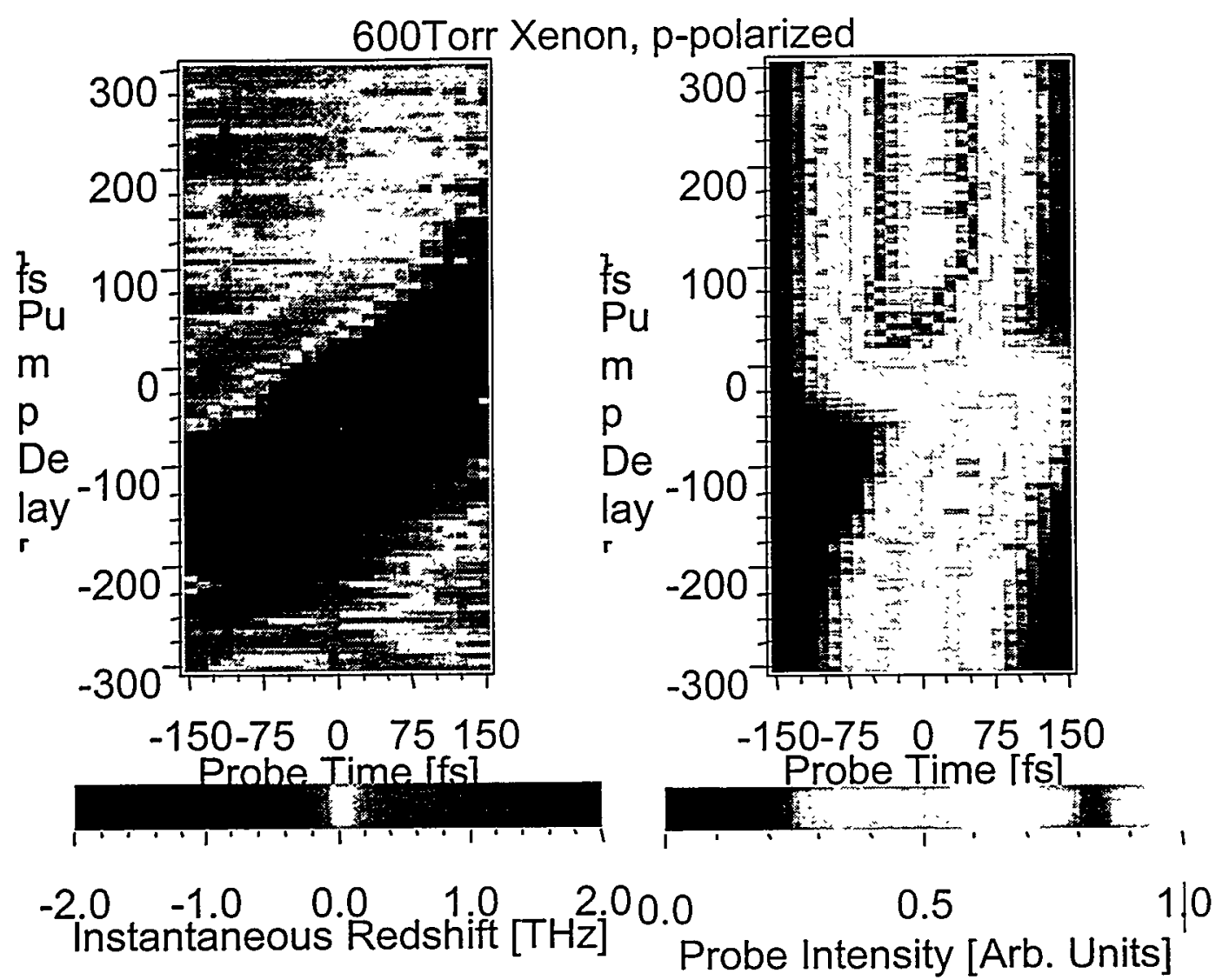

Figure 4. (a) Doubly time-resolved frequency shift (left) in $600 \mathrm{~T}$ Xenon with pump and probe orthogonally polarized. The large dark diagonal corresponds to a strong blueshift, while the upper, lighter diagonal corresponds to a weak redshift. (b) Time-resolved probe intensity profile (right). 


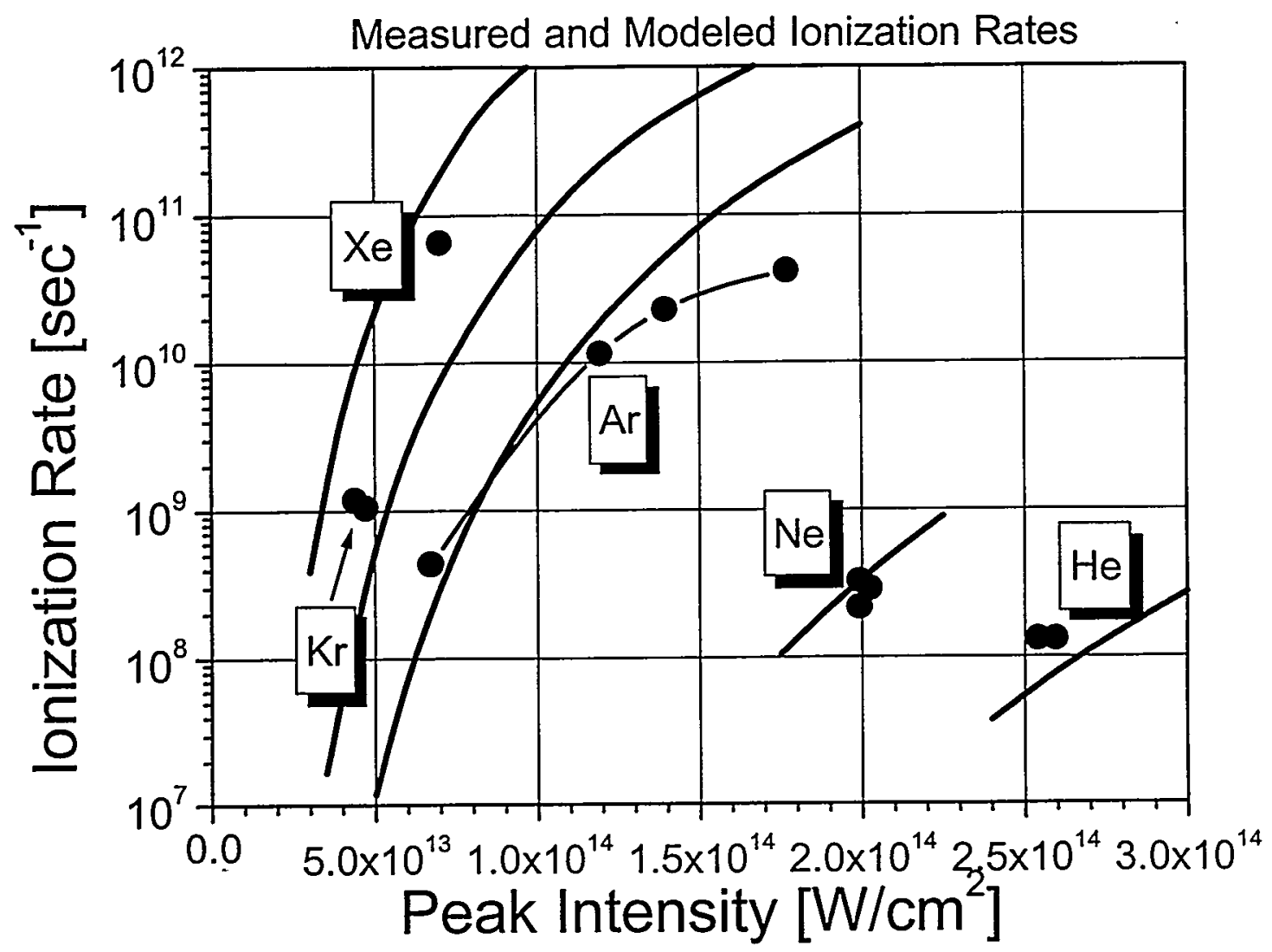

Figure 5. Measured and modeled ionization rates for the noble gases with the calculated rates obtained using a $1 \mathrm{D}$ plasma fluid model and tunneling theory ionization rates. 


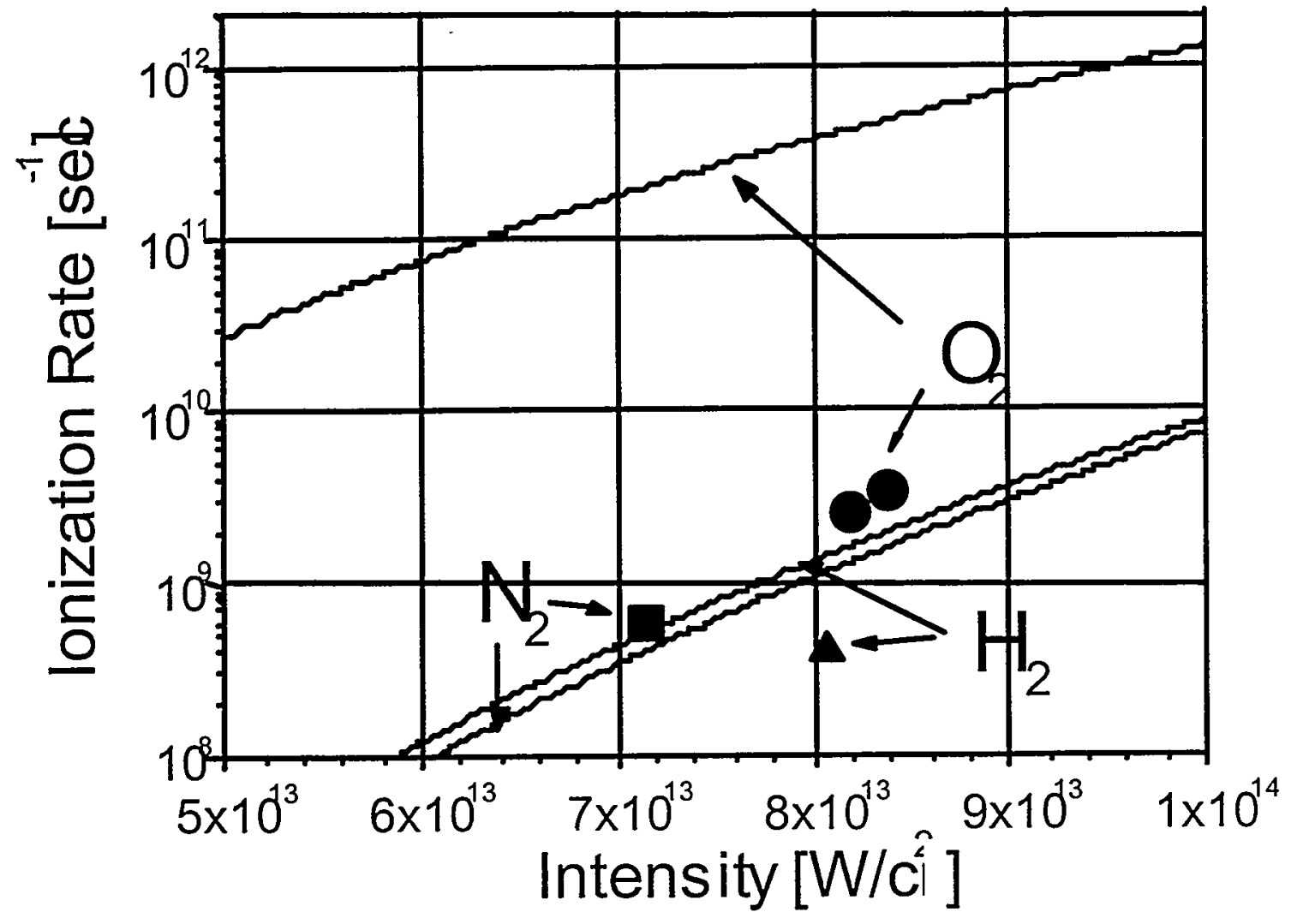

Figure 6. Measured and modeled ionization rates for $\mathrm{N}_{2}, \mathrm{O}_{2}$, and $\mathrm{H}_{2}$. 